\title{
On the global structure of distant galactic disks ${ }^{\star}$
}

\author{
V. P. Reshetnikov ${ }^{1,2,3}$, R.-J. Dettmar ${ }^{2}$, and F. Combes ${ }^{4}$ \\ 1 Astronomical Institute of St. Petersburg State University, 198504 St. Petersburg, Russia \\ 2 Astronomisches Institut der Ruhr-Universität Bochum, Universitätsstr. 150 NA7, 44780 Bochum, Germany \\ 3 Isaac Newton Institute of Chile, St. Petersburg Branch, Russia \\ ${ }^{4}$ LERMA, Observatoire de Paris, 61 Av. de l'Observatoire, 75014 Paris, France
}

Received 11 July 2002 / Accepted 3 December 2002

\begin{abstract}
Radial and vertical profiles are determined for a sample of 34 edge-on disk galaxies in the HDFs, selected for their apparent diameter larger than 1.3" and their unperturbed morphology. The thickness and flatness of their galactic disks are determined and discussed with regard to evolution with redshift. We find that sub- $L^{*}$ spiral galaxies with $z \sim 1$ have a relative thickness or flatness (characterized by $h_{z} / h$ the scaleheight to scalelength ratio) globally similar to those in the local Universe. A slight trend is however apparent, with the $h_{z} / h$ flatness ratio larger by a factor of $\sim 1.5$ in distant galaxies if compared to local samples. In absolute value, the disks are smaller than in present-day galaxies. About half of the $z \sim 1$ spiral disks show a non-exponential surface brightness distribution.
\end{abstract}

Key words. galaxies: evolution - galaxies: high-redshift - galaxies: structure

\section{Introduction}

A very fundamental characteristic of galactic disks is the flatness, defined by the ratio between exponential scalelengths in the vertical and radial direction $h_{z} / h$. The determination of the thickness of stellar disks is a difficult enterprise and has been determined mostly for edge-on galaxies, although attempts have been made in other cases, too (Ma et al. 1998). Typical values are found to be between 0.15 and 1 (van der Kruit \& Searle 1981, 1982; de Grijs 1998) with a systematic trend for galaxies to become thinner from $\mathrm{S} 0$ to $\mathrm{Sc}$ if a more complex multicomponent structure (thick/thin disk) is not taken into account. Stellar velocity dispersion measurements show that the dispersion tends to grow with total luminosity, and vary radially as the square root of surface density, so as to support a constant scaleheight (Bottema 1993). Recently Kregel et al. (2002) have shown that the flattening of disks increases with the amplitude of their maximum rotation, and their total HI mass.

This flatness is an important clue to the formation and evolution history of galaxies. Disk stars are thought to be formed out of a very thin gaseous disk. The observed structure then thickens due to scattering by local fluctuations of the gravitational field as caused, e.g., by giant molecular clouds or spiral structure in the disk. The velocity dispersion of the stars increases in both the radial and vertical direction by this diffusion through phase space (Wielen 1977;

Send offprint requests to: V. P. Reshetnikov,

e-mail: resh@astro.spbu.ru

* Figure 8 (Appendix) is only available in electronic form at http://www. edpsciences.org
Binney \& Lacey 1988). The disk may thicken more efficiently through interactions with small companions or minor mergers, as suggested by numerical simulations (Quinn et al. 1993; Walker et al. 1996; Velazquez \& White 1999). The thickening could amount to a factor of 1.5-2 and can be a constraint on the frequency of mergers if disks are observed today to be too thin (e.g. Toth \& Ostriker 1992). The existence of a thick stellar disk in our own Galaxy has been attributed to an old merger event (Robin et al. 1996; Dalcanton \& Bernstein 2002). However, other phenomena have to be taken into account since the thin disk could also be maintained through gas infall with subsequent star formation. Many observational facts strongly suggest a high gas infall rate (e.g. Toomre 1990; Sancisi et al. 1990; Jiang \& Binney 1999). Dynamics of galaxy disks, bar and spiral reformation constrain this rate such that galaxies may double their mass in less than 10 Gyrs (Bournaud \& Combes 2002; Block et al. 2002).

The influence of tidal interaction on the flatness of galaxy disks has been addressed by Reshetnikov et al. (1993) and Reshetnikov \& Combes (1997) who found in interacting galaxies the ratio $h_{z} / h$ to be a factor of two higher, due to a disk thicker in absolute values, but also shorter in radial dimensions. Schwarzkopf \& Dettmar (2000a, 2001) confirmed this trend on a larger sample, but essentially because of a larger absolute thickness of interacting disks. They also noted that tidally interacting galaxies are more frequently warped and that the thickening was more noticeable in the outer parts.

All these studies suggest that the relative flatness of a galaxy disk can vary by both effects during its evolution since 
the majority of galaxies have experienced interactions in the past. It appears that galaxies were dynamically "hotter" in the past (Abraham et al. 1999), which is seen in a smaller fraction of barred galaxies, and this could also have some consequences for the flattening. As the frequency of interactions/mergers strongly increases with redshift (e.g. Le Fèvre et al. 2000), it is interesting to trace the evolution of the disk flatness with time by studying photometrically distant galaxies with high spatial resolution. This is now possible in the Hubble Deep Fields HDF-N and HDF-S and we present below the results of such a study. Section 2 describes our sample and Sect. 3 gives the derived general characteristics of disks in terms of radial and vertical profiles. The last section discusses the main results on the evolution of the flatness for stellar disks.

Throughout the paper, we adopt a flat cosmology with $\Omega_{0}=1$ and $H_{0}=70 \mathrm{~km} \mathrm{~s}^{-1} \mathrm{Mpc}^{-1}$. (For a model with $\Omega_{m}=1 / 3, \Omega_{\Lambda}=2 / 3$ and $\Omega_{m}+\Omega_{\Lambda}=1$, linear sizes at $z=0.7-1$ will be $\approx 25 \%$ larger and absolute magnitudes are brigther by $\approx 0$. 45 .) If not differently specified, all magnitudes are expressed in the AB system (Oke 1974).

\section{The sample}

To study the structural parameters of distant edge-on spiral galaxies, we consider a sample of galaxies presented in Paper I (Reshetnikov et al. 2002). This sample includes 45 galaxies with a diameter larger than 1 .' 3 selected in the Hubble deep fields north and south. By applying a $V / V_{\max }$ completeness test we derived that the sample is statistically complete $-V / V_{\max }=$ $0.50 \pm 0.04$ (Paper I). From the total sample of 45 distant galaxies we rejected objects with strong overlaps or interactions (e.g. n1031, SB-WF-2353-1914/2340-1898) and objects with unusual morphology (for instance, the "tailed" galaxies n16, n632, n1027). Also, we excluded the galaxies n749 and SB-WF-3329-3173 since they seem to be deviating from the edge-on orientation. The final sample consists of 34 edge-on galaxies suitable for further analysis.

The mean isophotal diameter of the galaxies within $\mu\left(I_{814}\right)=26.0$ is $2^{\prime \prime} 3(14 \mathrm{kpc}$ at $z=0.9)$, the mean minor axis is $0 .^{\prime} 7$. The latter is about 5 times larger than the FWHM of the PSF and therefore the galaxies are sufficiently resolved to study the vertical surface brightness distribution.

The general characteristics of the sample galaxies are presented in Table 1. Figure 8 (see appendix) gives contour maps and radial major axis profiles of the objects.

\section{General characteristics of edge-on disks}

As judged from to the spectral energy distributions (FernándezSoto et al. 1999), late-type galaxies (Sbc/Irr) predominate in our sample (Table 1). The mean redshift of the sample and the median of the distribution are the same, $z \approx 0.9$. The mean rest-frame absolute magnitude of the galaxies is relatively low $-M(B) \approx-18^{\mathrm{m}}$ (Paper I). This is not unexpected since the Hubble deep fields have a very small coverage of only $\sim 2 \mathrm{Mpc}$ (comoving) at $z \sim 1$ each. At $z<1$, the expected number of bright $L^{*}$ galaxies in the two fields is only $\sim 20-60$ (Ferguson et al. 2000). Therefore, assuming random orientation
Table 1. Edge-on galaxies in the HDF-N and HDF-S.

\begin{tabular}{|c|c|c|c|c|}
\hline ID & $I_{814}$ & $b / a$ & $z$ & Type \\
\hline 15 & 24.70 & 0.28 & 0.45 & Scd \\
\hline 55 & 23.32 & 0.20 & 0.18 & Irr \\
\hline 120 & 24.27 & 0.24 & 1.60 & Irr \\
\hline 153 & 24.94 & 0.35 & 0.73 & Irr \\
\hline 273 & 22.47 & 0.45 & 0.905 & Ell \\
\hline 304 & 25.60 & 0.35 & 1.78 & Scd \\
\hline 450 & 24.25 & 0.30 & 0.70 & Irr \\
\hline 476 & 22.77 & 0.39 & 0.421 & Irr \\
\hline 506 & 24.47 & 0.25 & 0.751 & Irr \\
\hline 534 & 23.82 & 0.25 & 0.95 & $\mathrm{Sbc}$ \\
\hline 671 & 23.93 & 0.36 & 0.681 & Irr \\
\hline 716 & 22.61 & 0.30 & 0.944 & $\mathrm{Sbc}$ \\
\hline 727 & 23.93 & 0.33 & 0.904 & Scd \\
\hline 733 & 25.04 & 0.25 & 0.94 & Irr \\
\hline 774 & 21.22 & 0.28 & 0.485 & $\mathrm{Sbc}$ \\
\hline 805 & 25.33 & 0.30 & 0.66 & Irr \\
\hline 817 & 22.19 & 0.40 & 0.485 & $\mathrm{Sbc}$ \\
\hline 886 & 25.25 & 0.30 & 0.97 & Irr \\
\hline 888 & 23.70 & 0.30 & 0.559 & Irr \\
\hline 898 & 25.89 & 0.20 & 0.94 & Irr \\
\hline 899 & 22.94 & 0.20 & 0.564 & Scd \\
\hline 938 & 23.03 & 0.27 & 0.557 & Scd \\
\hline 979 & 23.45 & 0.27 & 0.517 & Scd \\
\hline S0564 & 25.27 & 0.35 & 1.02 & Irr \\
\hline S0566 & 25.67 & 0.30 & 2.16 & Scd \\
\hline S0578 & 23.16 & 0.22 & 0.98 & Scd \\
\hline S0747 & 25.31 & 0.24 & 0.98 & Irr \\
\hline S1085 & 25.51 & 0.29 & 1.13 & Irr \\
\hline S1404 & 23.09 & 0.20 & 0.50 & Sbc \\
\hline S2661 & 24.10 & 0.30 & 0.92 & Irr \\
\hline S2691 & 25.05 & 0.35 & 0.46 & Irr \\
\hline S3053 & 26.07 & 0.30 & 1.05 & Scd \\
\hline S3458 & 23.40 & 0.40 & 0.69 & Scd \\
\hline S3685 & 26.63 & 0.35 & 2.23 & SB1 \\
\hline
\end{tabular}

The columns are: object identification (Paper I); $I_{814}$ magnitude in the AB photometric system according to Fernández-Soto et al. (1999) (aperture magnitude corrected for the effect of neighboring objects and the flux lost in pixels outside the aperture); apparent axial ratio (from ellipse-fitting of the outer contours); redshift (spectroscopic redshifts are with three numbers, photometric redshifts obtained as the mean of redshifts presented by Fernández-Soto et al. 1999 and by Fontana et al. 2000); best-fit spectral type of galaxy (Fernández-Soto et al. 1999).

of galaxies, we can expect to find a few highly inclined bright spirals at $z<1$.

Two of our edge-on galaxies are possible sources of submm (SCUBA) radiation (Serjeant et al. 2002). These are the objects 304 and 774 in Table 1.

The mean observed axial ratio of the sample objects is 0.3 (Paper I, Table 1), a value exceeding the $b / a$ ratio for local edge-on late-type galaxies. Possible reasons are a large intrinsic thickness of distant objects and also the influence of the pointspread function (PSF) on the apparent thickness of the small $\left(\sim 2^{\prime \prime}\right)$ HDF galaxies.

The largest uncertaintiy arises from the range of galaxy inclinations in our sample since we do not know the true intrinsic flatnesses of the sample objects and we also cannot use 
a standard approach, e.g., on the basis of the Hubble formula or from dust lane geometry. We selected our sample on the basis of careful visual inspection of the HDF frames. Secondary inclination indicators (asymmetry of the minor axis profiles, apparent shift of the nucleus location from the symmetry plane) are in the same range as in local samples of highly inclined spirals (e.g. de Grijs \& van der Kruit 1996; Schwarzkopf \& Dettmar 2000a). Also, comparison with results of realistic numerical models for inclined spirals (e.g. Byun et al. 1994) indicates that most of our sample objects are within $5^{\circ}$ of edge-on orientation. Such a moderate deviation from edge-on orientation does not significantly change the slope of the vertical surface brightness distribution (van der Kruit \& Searle 1981; Barteldrees \& Dettmar 1994).

\subsection{Optical colors}

Figure 1 compares the observed colors of the 14 sample objects with spectroscopic redshifts and of nearly face-on spirals (randomly selected from the HDF-N) as a function of redshift. Both subsamples demonstrate the same trend of colors with $z$ with a somewhat larger scatter for the edge-on disks. In the redshift interval $z=0.4-1$ the edge-on disks show a hint of reddening in comparison with the face-on spirals: $E_{u-b}=+0.25 \pm 0.18$ and $E_{b-v}=+0.23 \pm 0.13$. These estimates correspond to inclination corrections for local spiral galaxies (e.g. de Vaucouleurs et al. 1991).

The predictions of apparent color evolution for two models of galaxy formation are shown in Fig. 1 according to Westera et al. (2002). The first model is characterized by a slowly growing dark halo with a continuous infall of gas and dark matter (accretion model, dashed line). The second one is a classical collapse model in a dark matter halo (collapse model, dotted line). Obviously, both models provide a satisfactory description of general observational trends of colors with redshift.

\subsection{Major axis profiles}

To investigate the global structure of the sample objects we adopted as a fitting model the simple standard double exponential disk: $L(r, z)=L_{0} \exp \left(-\frac{r}{h}-\frac{|z|}{h}\right)$, where $h$ and $h_{z}$ are the scalelength and scaleheight, respectively. This simple model gives a reasonable description of the structure for most local disks, even in edge-on orientation (e.g. Misiriotis et al. 2000). Comparison with other vertical models for disks (for instance, with " $\operatorname{sech}^{2}\left(z / z_{0}\right)$ " or " $\operatorname{sech}\left(z / z_{0}^{\prime}\right)$ " distributions) can be done via $h_{z}=z_{0} / 2=z_{0}^{\prime} / \sqrt{2}$.

\subsubsection{Exponential profiles}

Figure 8 presents the major axis photometric profiles of all galaxies in the $I_{814}$ passband. The profiles show a large diversity in their shapes. Trying to fit the surface brighness distributions by an exponential law, we find that only 19 or $56 \% \pm 7 \%$ of the disks are reasonably well described by exponential-like profiles.
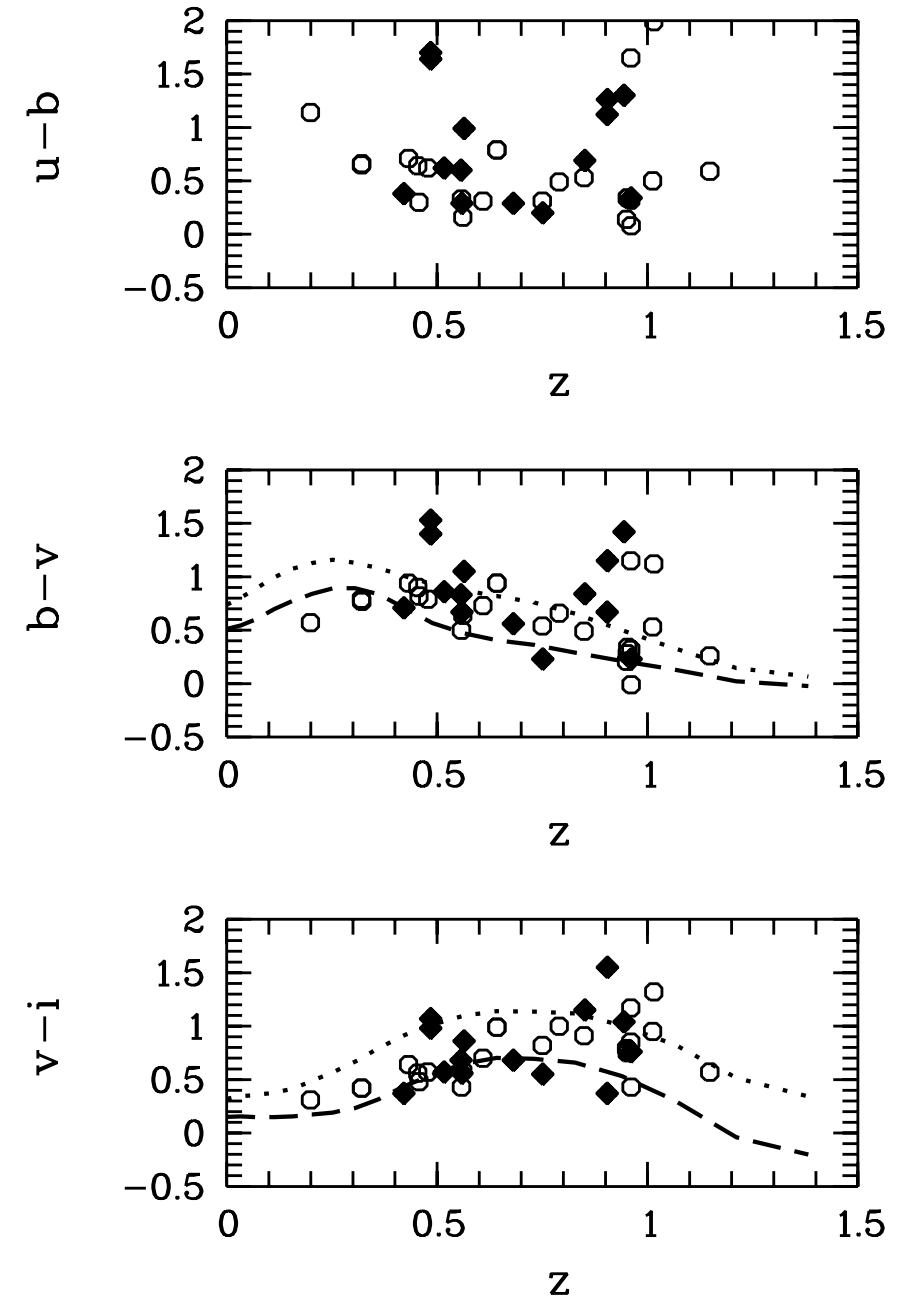

Fig. 1. Observed color - spectroscopic redshift dependence for the HDF edge-on galaxies (solid rhombs) and for face-on spirals (open circles). The $u-b, b-v$, and $v-i$ corresponding to $U_{300}-B_{450}$, $B_{450}-V_{606}$, and $V_{606}-I_{814}$ colors (Williams et al. 1996). The lines show predicted color evolution as a function of redshift for two models of disk galaxy formation - the accretion (dashed line) and the collapse (dotted line) models (Westera et al. 2002).

The parameters for the exponential disks are presented in Table 2. Typical errors for the scalelength values are $\approx 10 \%$. The errors of scaleheights are presented in Table 2 and they are not just the formal errors of the least-squares fit, rather they were estimated by comparing results from four independent fits (see Sect. 3.3). To check the reliability of the exponential model to describe the global photometric structure of the studied galaxies, we have calculated total luminosities as $L_{\text {exp }}=2 \pi I_{0} h^{2} b / a$, where $I_{0}=\operatorname{dex}\left(-0.4 \mu_{0}^{I}\right)$ (Table 2) and $b / a$ is the apparent axial ratio (Table 1$)$. We found that the mean difference of observed and model magnitudes is $\left\langle I_{814}-I_{\text {exp }}\right\rangle=$ $+0.31 \pm 0.12$. This small difference can be attributed to the infinite extrapolation for the model magnitudes.

The mean observational characteristics of the exponential disks are $\left\langle\mu_{0}\left(I_{814}\right)\right\rangle=(22.99 \pm 1.16)^{\mathrm{mag}} / \square^{\prime \prime},\langle h\rangle=0{ }^{\prime} 43 \pm 00^{\prime} 11$, $\langle z\rangle=1.0 \pm 0.5$. The observed values of $\mu_{0}\left(I_{814}\right)$ (uncorrected for any inclination effects) have been converted to a rest-frame $B$ by applying the cosmological dimming term and a k-correction 
color term for Irr galaxies (Lilly et al. 1998; Lilly et al. 1995; Paper I). The resulting mean characteristics of the central surface brightness and of the scale length (after a small correction for the PSF - see Sect. 3.3) are $\left\langle\mu_{0}(B)\right\rangle=(20.7 \pm 1.3)^{\mathrm{mag}} / \square^{\prime \prime}$, $\langle h\rangle=2.3 \pm 0.7 \mathrm{kpc}$. For the disks with $z \leq 1$ (14 objects), the central surface brightness is $(21.05 \pm 1.0)^{\mathrm{mag}} / \square^{\prime \prime}$. Therefore, distant edge-on disks demonstrate somewhat brighter central surface brightnesses in comparison to nearby "Freeman" disks for which $\mu_{0}(B)=21.5^{\mathrm{mag}} / \square^{\prime \prime}$ in the AB system (Freeman 1970). However, this difference is negligible within the quoted scatter. Note also that our derived value of the mean central surface brightness is in good agreement with results for local samples of edge-on galaxies: $\left\langle\mu_{0}(B)\right\rangle=(21.0 \pm 0.6)^{\mathrm{mag}} / \square^{\prime \prime}$ (28 members of interacting systems, Reshetnikov \& Combes 1997), $\left\langle\mu_{0}(B)\right\rangle=(21.0 \pm 1.1)^{\mathrm{mag}} / \square^{\prime \prime}$ (45 normal galaxies, de Grijs 1998).

As a first order approximation, let us assume that the galaxy centers are optically thick, or close to that, and so the central surface brightness does not depend strongly on the inclination of the disk. This is a quite realistic approximation since at redshift $z=0.9$ the filter $I_{814}$ corresponds approximately to filter $B$ $(8140 \AA:(1+0.9)=4280 \AA)$ and the central regions of local spirals are not optically thin in the blue spectral region. There are several lines of arguments - discussed in the following - in favour of such a conclusion.

The only direct and model-independent way to study the transparency of the centers of galaxies is the chance superposition of galaxies. In the innermost few hundred parsecs of NGC 3314A (a sub- $L^{*}$ Sc spiral galaxy) even the most transparent regions between dust lanes show an extinction of $A_{B} \approx 7^{\mathrm{m}}$ (Keel \& White 2001). The disk of NGC 3314A is inclined by $i=41^{\circ}$, so this galaxy is far from edge-on orientation.

The same conclusion can be derived from statistical arguments. For instance, Giovanelli et al. (1995), using a sample of more than $1700 \mathrm{Sc}$ galaxies with $I$-band photometry, found only a weak dependence of the central surface brightnesses of the disks on inclination: $\mu_{0}(I)$ brightens by about $0.3-0.4$ mag between the face-on and edge-on aspects. In the $B$ band the dependence must be even much weaker. Also, with regard to distant galaxies, Lilly et al. (1998) did not find any significant correlation between $\mu_{0}(B)$ and disk axial ratio for spirals with $z=0.2-1$.

Numerical simulations of the radiative transfer in dusty disks show that even a small optical depth can significantly reduce the expected projection effect on the central surface brightness, especially in the $B$ band (Byun et al. 1994).

Therefore, assuming that the central surface brightness shows only a weak (or negligible) dependence on the galaxy inclination $\left(\Delta \mu_{0}(B)=(0-0.5)^{\mathrm{mag}} / \square^{\prime \prime}\right)$ we can estimate an average "face-on" absolute magnitude of the disks as $\langle M(B)\rangle=$ $\left\langle\mu_{0}(B)\right\rangle-5 \log \langle h\rangle-38.57=-19^{\mathrm{m}} 6 \ldots-19^{\mathrm{m}} 1$. (Let us note also that in edge-on orientation the presence of dust makes the scalelength appear larger than in the face-on case (e.g. Byun et al. 1994) and this can reduce the estimated face-on luminosity.) The observational value for edge-on disks is -18 m $^{\mathrm{m}} 1$ or $1.0 \ldots 1.5$ fainter. The value of dimming $(1.0 \ldots 1.5)$ is consistent within uncertaintes with the value of global extinction for spirals (e.g. de Vaucouleurs et al. 1991; Tully et al. 1998).

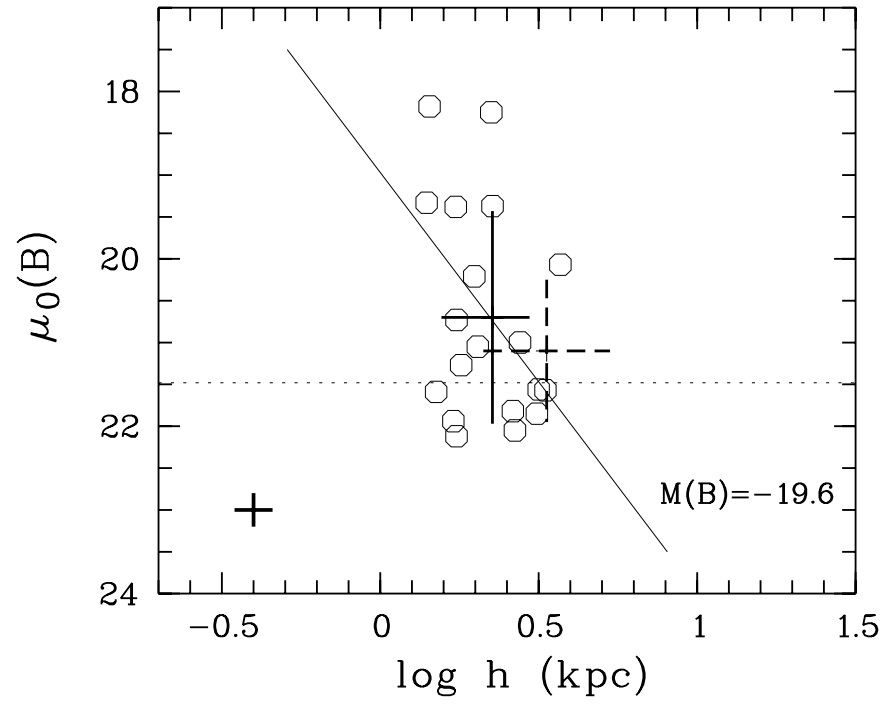

Fig. 2. $\mu_{0}(B)-h$ plane for edge-on galaxies. Typical error of the data is shown by a small cross at bottom left. The dotted line shows the Freeman law, the solid line is the line of constant disk luminosity. The solid cross gives the mean characteristics of our edge-on disks and the dashed cross is the mean for the sample of 917 local late type spirals with $M(I) \geq-22^{m}$ from Byun (1992).

Table 2 presents also the $h$ values in the $V_{606}$ passband. The mean scale length ratio is $\left\langle h\left(I_{814}\right) / h\left(V_{606}\right)\right\rangle=1.04 \pm 0.13$. (In the rest-frame this ratio resembles $h(B) / h(U)$.) Although we did not find any significant color dependence as expected from the modeling by de Jong (1996) for these filters, we cannot draw any firm conclusions due to the small-number statistics.

In Fig. 2 we consider the $\mu_{0}-h$ plane. The dashed cross in the figure shows the mean parameters of $917 \mathrm{Sb}-\mathrm{Sd}$ spiral galaxies from Byun's (1992) thesis (converted to $B_{A B}$ ). The sample of Byun is angular diameter-limited (like ours) and contains galaxies with angular diameter $d \geq 1$ :7. The mean absolute magnitude of this sample of local galaxies is $\langle M(I)\rangle=-20^{\mathrm{m}} .4$, or $\langle M(B)\rangle \approx-18^{\mathrm{m}} .7$ (adopting $B-V=+1.7$ for the disks of galaxies, de Jong 1996). Therefore, in edge-on orientation the local galaxies will show about the same luminosities as our sample galaxies. As one can see in the figure, the mean characteristics of nearby and distant sub- $L^{*}$ disks are close (within quoted uncertaintes) but $z \sim 1$ galaxies show a small displacement to higher values of surface brightness and smaller sizes. The shift to smaller values of $h$ can be even larger due to the above mentioned effect of an apparent increase of scalelength in edge-on orientation.

At least three objects (n15, n805, and n898) can be considered as low surface brightness galaxies. These three galaxies show the rest-frame central surface brightness $\mu_{0}(B) \approx 22$ and are intrinsically faint $\left(M(B) \geq-17^{\mathrm{m}}\right)$. Adopting small or neglible internal absorption for such galaxies (e.g. Tully et al. 1998) and converting the observational values of $\mu_{0}(B)$ to faceon orientation, we obtain $\mu_{0}^{0}(B) \approx 23.51-24^{\mathrm{mag}} / \square^{\prime \prime}$, typical for local low surface brightness galaxies (e.g. UGC 7321 Matthews \& Wood 2001). 
Table 2. Characteristics of galaxies with exponential-like surface brightness distribution.

\begin{tabular}{|c|c|c|c|c|c|c|c|}
\hline ID & $\mu_{0}^{I}$ & $\begin{array}{l}h^{I} \\
\left({ }^{\prime \prime}\right)\end{array}$ & $\mu_{0}^{V}$ & $\begin{array}{l}h^{V} \\
\left({ }^{\prime \prime}\right)\end{array}$ & $h_{z}^{I} / h^{I}$ & $\begin{array}{c}h^{I} \\
(\mathrm{kpc})\end{array}$ & $\begin{array}{c}h_{z}^{I} \pm \sigma_{h_{z}} \\
\quad(\mathrm{kpc})\end{array}$ \\
\hline 15 & 22.94 & 0.38 & 23.27 & 0.46 & 0.18 & 1.70 & 0.310 .06 \\
\hline 153 & 23.17 & 0.29 & 23.69 & 0.28 & 0.21 & 1.50 & 0.320 .10 \\
\hline 304 & 23.70 & 0.32 & 24.05 & 0.35 & 0.27 & 1.73 & $\begin{array}{lll}0.47 & 0.25\end{array}$ \\
\hline 450 & 23.34 & 0.48 & 23.79 & 0.48 & 0.33 & 2.62 & $0.86 \quad 0.34$ \\
\hline 476 & 21.67 & 0.40 & 22.22 & 0.46 & 0.27 & 1.74 & $\begin{array}{lll}0.47 & 0.09\end{array}$ \\
\hline 671 & 22.54 & 0.39 & 23.25 & 0.37 & 0.23 & 2.03 & $0.47 \quad 0.16$ \\
\hline 727 & 22.28 & 0.36 & 22.50 & 0.34 & 0.18 & 1.98 & 0.360 .06 \\
\hline 733 & 23.76 & 0.59 & 24.54 & 0.70 & 0.17 & 3.32 & 0.560 .11 \\
\hline 774 & 20.42 & 0.31 & 21.57 & 0.32 & 0.19 & 1.40 & 0.270 .03 \\
\hline 805 & 23.56 & 0.34 & 24.03 & 0.35 & 0.14 & 1.74 & $0.24 \quad 0.07$ \\
\hline 817 & 20.46 & 0.47 & 21.55 & 0.47 & 0.4 & 2.26 & $0.90 \quad 0.21$ \\
\hline 888 & 22.52 & 0.37 & 23.03 & 0.35 & 0.24 & 1.80 & $0.43 \quad 0.07$ \\
\hline 898 & 24.24 & 0.47 & & & 0.17 & 2.66 & $\begin{array}{ll}0.45 & 0.09\end{array}$ \\
\hline S0566 & 24.00 & 0.42 & 24.43 & 0.52 & 0.18 & 2.24 & $0.40 \quad 0.10$ \\
\hline S0578 & 22.36 & 0.64 & & & 0.25 & 3.70 & $\begin{array}{ll}0.93 & 0.20\end{array}$ \\
\hline S0747 & 23.85 & 0.57 & 24.36 & 0.56 & 0.13 & 3.16 & $0.41 \quad 0.08$ \\
\hline S1085 & 23.69 & 0.51 & 24.00 & 0.41 & 0.10 & 2.76 & $0.28 \quad 0.03$ \\
\hline S3053 & 24.38 & 0.57 & 25.4 & 0.7 & 0.11 & 3.12 & $0.34 \quad 0.14$ \\
\hline S3685 & 24.00 & 0.28 & 23.86 & 0.24 & 0.22 & 1.43 & $\begin{array}{ll}0.31 & 0.11\end{array}$ \\
\hline
\end{tabular}

The columns are: galaxy identification; central surface brightness and exponential scale length in arcsec ( $I_{814}$ passband); the same for the $V_{606}$ filter; scale height to scale length ratio corrected for the PSF; final values of scale length and scale height in kpc.

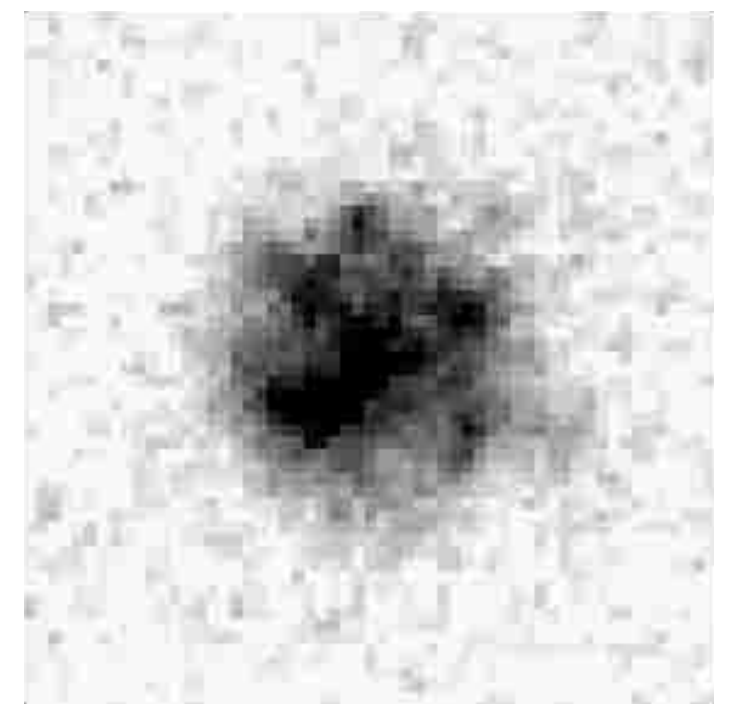

Fig. 3. Reproduction of the HDF-N galaxy $866\left(2{ }^{\prime \prime} 3 \times 22^{\prime \prime} 3\right)$ in the $I_{814}$ passband.

\subsubsection{Non-exponential profiles}

15 of 34 disks $(44 \% \pm 5 \%)$ show "ridge"-like profiles, with several peaks and are often very asymmetric (see, e.g., n55, n506, or n886 in Fig. 8). Therefore, roughly half of $z \sim 1$ disks are not exponential. This fraction may be even larger due to preselection of the sample (Sect. 2). This strongly contrasts with the local Universe and supports a late formation of disks (e.g. Mo et al. 1998, see van den Bergh et al. 2000 about galaxy morphology evolution with redshift).

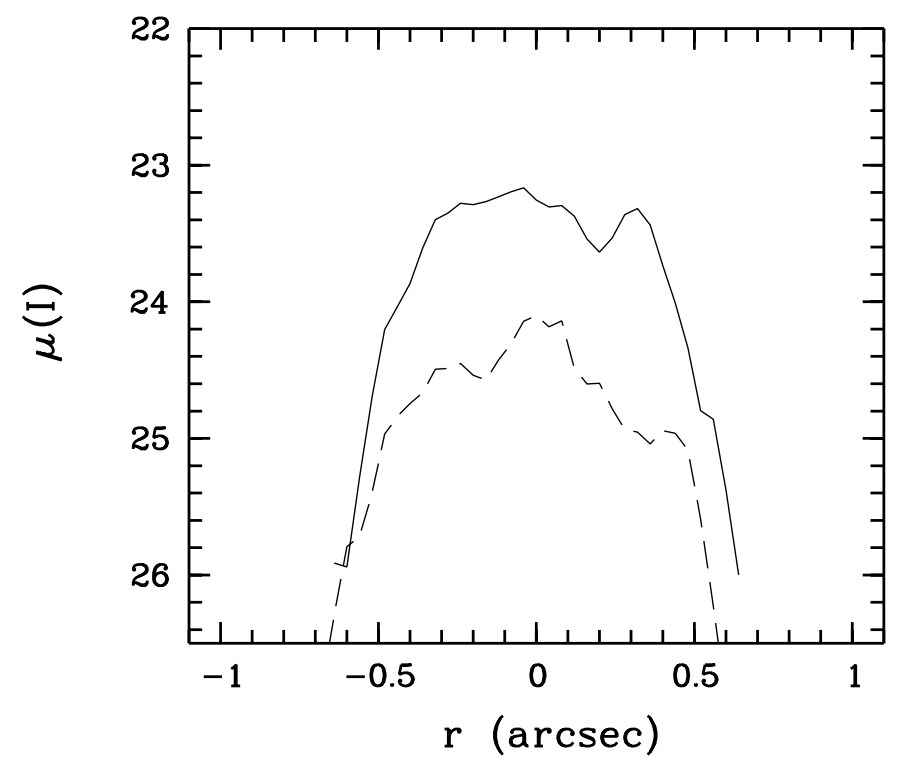

Fig. 4. Photometric profiles of HDF-N 866. Solid line $-\mathrm{PA}=125^{\circ}$, dashed line (the profile shifted down by $1^{\mathrm{m}}$ ) $-\mathrm{PA}=35^{\circ}$. The PA orientation is according to Fig. 3 (from top to the left).

To understand the origin of non-exponential brightness distributions, we have looked for possible counterparts for such objects among face-on galaxies in the deep fields. One of the best examples is the HDF-N galaxy 866 (2-153.0 in Williams et al. 1996) with the photometric redshift $0.96, I_{814}=23.6$ and the spectral type Scd (Fernández-Soto et al. 1999). The observed characteristics of this galaxy are close to those for the sample edge-on objects. This galaxy exhibits an irregular patchy disk with a low-contrast nucleus and embedded bright 
knots (five at least - see Fig. 3) and no spiral structure has developed yet. Figure 4 shows two photometric profiles of the galaxy which resemble the "ridge-like" profiles of many of our edge-on disks mentioned above. The galaxy is very similar in optical morphology to the peculiar galaxy H36490_1221 discussed in van den Bergh et al. (2001). Those authors suggest that such objects represent proto-late-type galaxies. Therefore, we can speculate that about half of our sample objects are young disks still in the formation process which will in their future evolution relax to an exponential distribution.

To check the frequency of non-exponential profiles among less distant spirals we selected in two deep fields all bright $\left(I_{814}<22^{m}\right)$ and non-edge-on galaxies of Sbc-Irr types. In total, we selected 17 galaxies with mean redshift $\langle z\rangle \approx 0.5$ and absolute magnitude $\langle M(B)\rangle \approx-20^{m}$. Almost one third $(5 / 17)$ of these objects show strongly non-exponential surface brightness distributions (work in preparation). The rest of the objects often demonstrate significant $(\sim 0.5)$ deviations from the exponent. Therefore, non-exponential density distributions of galaxy disks are indeed very frequent at $z>0$.

\subsection{Minor axis profiles}

The typical scalelengths of our objects are $\approx 0 .{ }^{\prime} 3-0 . ' 6$ (Table 2 ). Therefore, $h_{z}$ values are expected to be $\leq 0$.' 1 or below the PSF width of $F W H M$ (PSF) $=0$ '. 14. It is important to note that $h_{z}$ values are characteristics of the surface brightness gradients and are not linear sizes of any structures. To determine true scaleheights, we must study the effects of the HDF's PSF on the surface brightness distribution of edge-on galaxies.

We have created a grid of double exponential disk models with various input parameters in the region $h=0.3-0 . ' 6$ and $h_{z} / h$ from 0.10 to 0.35 . Then we prepared an empirical PSF by averaging several stellar images from the $I_{814}$-band frames of the HDF and convolved the model disks with the PSF. Comparing input and output disk characteristics, we determined empirical corrections for the observed parameters. The output parameters were estimated from the analysis of major and minor axes cuts of the model images. Finally, we found a strong influence of the PSF on the vertical scale of a disk. Also, we noted a small (within 10\%) overestimation of the scale length values due to the PSF.

To present the influence of the PSF more clearly, we show in Fig. 5 the dependence of vertical stretching due to the PSF on the intrinsic flattening $\left(h_{z} / h\right)$ of the model (the factor $\mathrm{S}$ gives the ratio of observed and true values of flattening). It is evident that intrinsically small $(h \approx 0 ! 3)$ and thin $\left(h_{z} / h \approx 0.1\right)$ galaxies become more than 2 times thicker after application of the PSF. Therefore, we cannot expect to find very thin galaxies among small and distant HDF objects.

We excluded the central regions of the galaxies from the study in order to avoid any possible contribution of the bulges. (Although we do not see clear signs of bulges present in most galaxies - see Fig. 8). In order to explore the vertical structure of 19 exponential objects we examined one-dimensional cuts extracted at $\pm(0.5-1) h$ in the galactocentric distance, avoiding the faintest outer regions of the objects. To minimize the

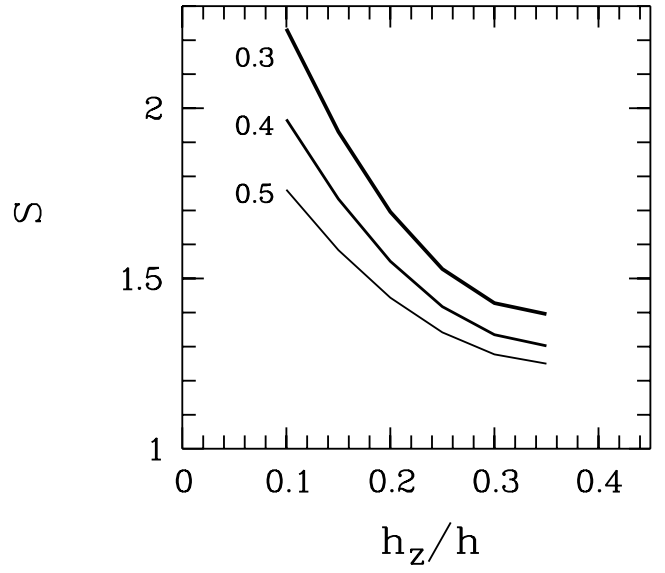

Fig. 5. Dependence of the "stretching" factor S due to the PSF on the true scaleheight to scalelength ratio $h_{z} / h$. The lines marked as $0.3,0.4$, 0.5 correspond to models with $h=0,3,0{ }^{\prime} 4,0,5$.

influence of dust, we excluded the 1-2 central pixels of the cuts. In the analysis, we distinguished between the different sides of the galaxy planes and obtained the mean value of $h_{z}$ for each galaxy from 4 independent estimates. The mean observational (uncorrected for the PSF influence) scale height for the sample objects is $00^{\prime} 13$, the mean dispersion $\sigma_{h_{z}}=00^{\prime} 03$. This value of dispersion (23\%) characterises the combined effect of the intrinsic variations of vertical scales and of errors in scale estimates.

The results of the analysis (corrected for the PSF) are given in the last three columns of Table 2 . The mean relative thickness of the galaxies reduces after the PSF correction from $\left\langle h_{z} / h\right\rangle_{\mathrm{obs}}=0.31 \pm 0.09$ to $\left\langle h_{z} / h\right\rangle=0.21 \pm 0.08$.

\subsubsection{Vertical scale}

The mean value of the vertical scale for distant edge-on galaxies is $\left\langle h_{z}\right\rangle=0.46 \pm 0.21 \mathrm{kpc}$ or $\left\langle z_{0}\right\rangle \approx 0.9 \mathrm{kpc}$. The obtained mean value is unbiased since we work with a statistically complete sample of galaxies with angular diameter larger than 1.'3 (Sect. 2, Paper I). These values are typical for normal spiral disks at $z \approx 0$ (e.g. Reshetnikov \& Combes 1997; Schwarzkopf \& Dettmar 2000a). However, the direct comparison of local and distant samples must be carried out with caution due to very different selection effects. For instance, distant galaxies are sub- $L^{*}$ and relatively compact while the local samples usually contain brighter and larger disk galaxies (see Table 2 in Reshetnikov \& Combes 1997).

In order to avoid (at least partially) the selection effects, we decided to compare our sample to local galaxies of the same size. Figure 6a shows the dependence of scaleheight on scalelength for 38 edge-on spiral galaxies $(T>0)$ in the $I$ passband according to de Grijs (1998). He adopted an exponential model for the observational brightness distribution and considered one-dimensional photometric profiles so we can directly compare our results. The mean regression for local galaxies predicts that a galaxy with $h=2.3 \mathrm{kpc}$ must have $h_{z}=$ $0.3 \mathrm{kpc}$. Therefore distant galaxies are thicker by a factor 1.5 on 

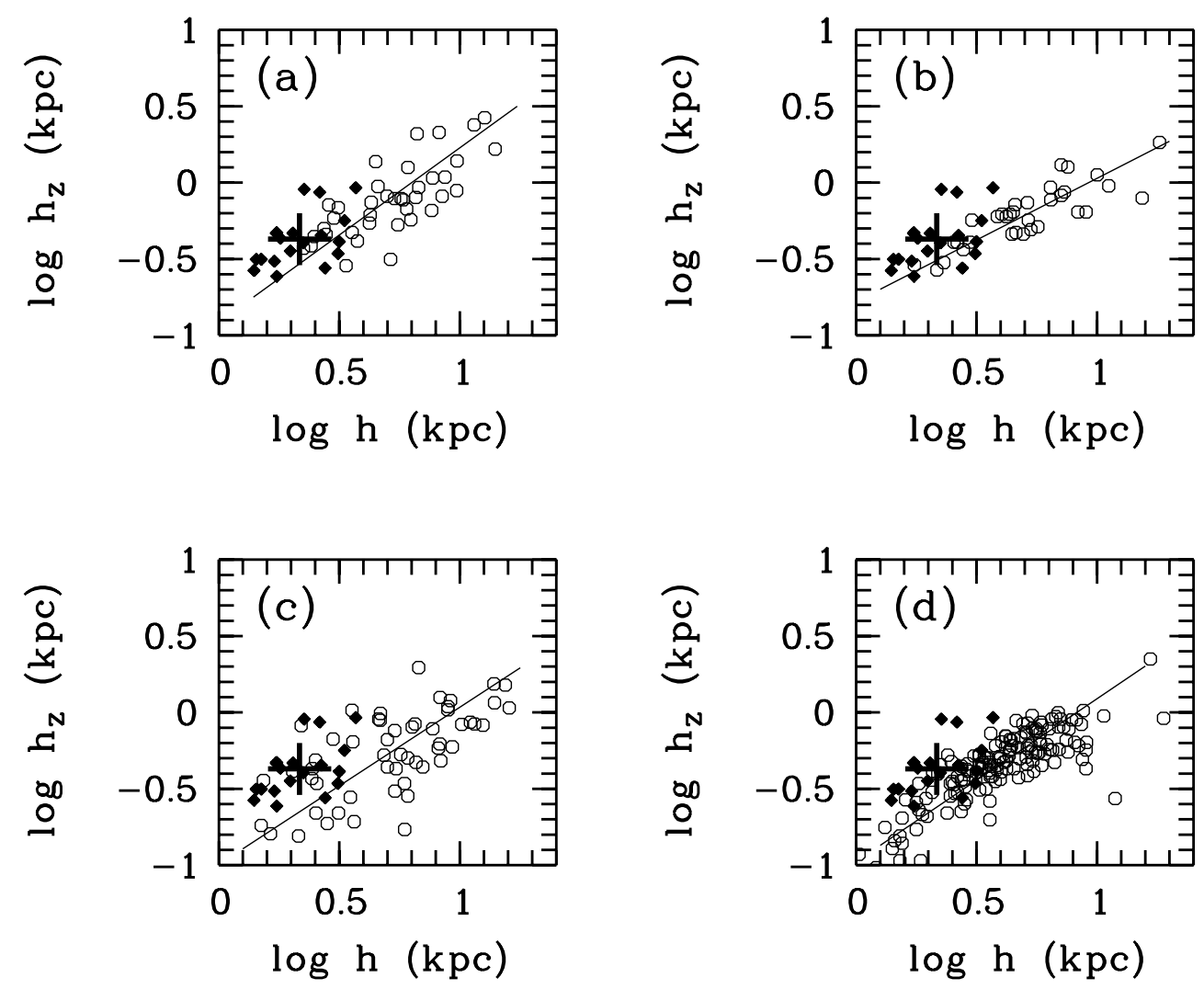

Fig. 6. Scalelength-scaleheight relation for local spirals (open circles; a) de Grijs 1998, b) Kregel et al. 2002, c) Schwarzkopf \& Dettmar 2000a, d) Bizyaev \& Mitronova 2002) and for our distant galaxies (solid rhombs). The solid lines show mean regressions for the nearby samples, the cross represents the average characteristics of distant galaxies and a $1 \sigma$ scatter is indicated.

average. (In the non-zero $\Lambda$ cosmology with $\Omega_{m}=1 / 3, \Omega_{\Lambda}=$ $2 / 3$ the relative thickening stays the same.)

Figure $6 \mathrm{~b}$ presents the results of a two-dimensional decomposition in the $I$ passband of 34 edge-on spirals from the de Grijs (1998) sample recently presented by Kregel et al. (2002). The fitting model assumes a transparent disk (this is very questionable for our sample objects) and includes the lineof-sight integration of the luminosity distribution. Comparison of two methods shows that a one-dimensional method can overestimate the scalelengths by about $20 \%$ (Fig. 3 in Kregel et al. 2002). However, a direct comparison of $h$ values shows that $h($ de Grijs $) / h$ (Kregel et al. $)=0.93 \pm 0.20$. A comparison of scaleheight values obtained by the two methods leads to a ratio $h_{z}($ de Grijs $) / h_{z}$ (Kregel et al. $)=1.13 \pm 0.22$. Strictly speaking, both methods give a statistically insignificant difference. The Kregel et al. (2002) analysis results in a $\sim 1.4$ thickening of distant disks.

In Fig. 6c we compare the Schwarzkopf \& Dettmar (2000a) sample of non-interacting galaxies (the data in the Johnson $R$ or Thuan \& Gunn $r$ filters). The fitting model avoids the central dust lane in the line-of-sight integration. In this case the thickening factor increases to 1.9.

The last sample (Fig. 6d) includes 153 edge-on spiral galaxy with published photometric parameters in the $K_{\mathrm{s}}$ band (Bizyaev \& Mitronova 2002). The fitting model assumes a transparent disk. This work gives about a 1.8 -fold thickening of distant galaxies.

\subsection{2. $h / h_{z}$ ratio}

The relative thickness $-h / h_{z}-$ gives more reliable information about the possible thickening. For our sample we have $\left\langle h / h_{z}\right\rangle=$ $5.4 \pm 2.0$, or, excluding one object for being most likely not exactly an edge-on galaxy (n817), $5.55 \pm 1.9$. Scaling this in terms of $z_{0}$, we obtain $\left\langle h / z_{0}\right\rangle=2.7-2.8$, with a median value of 2.63. The disks of local late-type galaxies are distributed over a wider range with the mean $h / z_{0}$ of $\approx 5$ (see Table 2 in Reshetnikov \& Combes 1997). Moreover, disks of more latetype and gas-rich galaxies are thinner (Reshetnikov \& Combes 1997; de Grijs 1998; Schwarzkopf \& Dettmar 2000a). For $\mathrm{Sc} / \mathrm{Sd}$ disks Schwarzkopf \& Dettmar (2000a) give $h / z_{0} \approx 8 \pm 2$. De Grijs (1998) and Kregel et al. (2002) give somewhat smaller ratios of 3.7 and 4.3, respectively. The Bizyaev \& Mitronova (2002) sample shows the mean ratio of $h / z_{0}=4.8$.

In the previous discussion we neglected the possible dependence of scalelength on the passband. It is known (e.g. Table 7 in de Grijs 1998) that the $h$ value increases to the blue spectral region. The ratio of scalelength values in the $B$ and $K$ passbands can reach $\approx 1.5$, and $h(B) / h(I) \approx 1.3$ (de Grijs 1998). This effect, however, would only strengthen the thickness difference in the samples of local and $z \sim 1$ galaxies.

Therefore, $z \sim 1$ disks are definitely thicker in comparison to local galaxies. The factor for the vertical thickening $(\geq 1.5)$ is approximately the same as for $z \approx 0$ interacting spiral galaxies ( 1.5-2: Reshetnikov \& Combes 1997; Schwarzkopf \& Dettmar 2000a). 


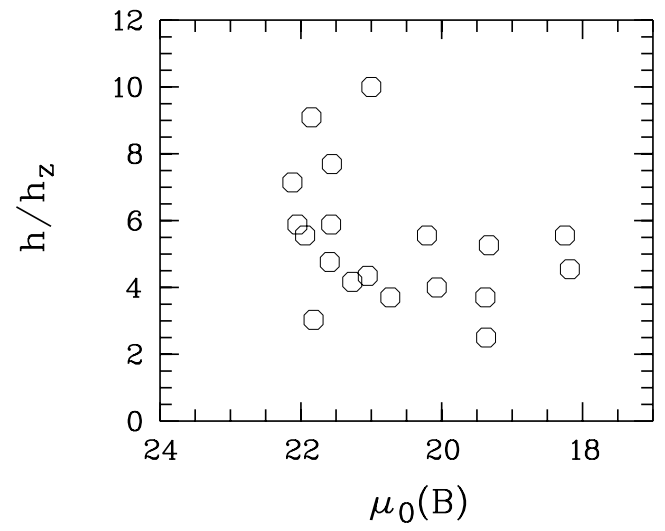

Fig. 7. Dependence of the relative thickness of distant galaxies on their rest-frame central surface brightness.

The dependence of $h / h_{z}$ values on the rest-frame central surface brightness is shown in Fig. 7. Thin disks are present for $\mu_{0}(B) \geq 21^{\mathrm{mag}} / \square^{\prime \prime}$, while among bright objects $\left(\mu_{0}(B)<\right.$ $\left.21^{\mathrm{mag}} / \square^{\prime \prime}\right)$ we observe only thick disks with $h / z_{0} \approx 2.2$. Taking into account that the $B$-band surface brightness characterises the current rate of star formation, one can speculate that enhanced star-formation and enhanced thickness are the results of ongoing interactions and mergings. The observed morphology of galaxies does not contradict such a suggestion (Fig. 8).

\subsection{Vertical scale for non-exponential galaxies}

We examined the vertical profiles for 15 non-exponential galaxies at distances where the surface brightness drops by $1^{\mathrm{m}}$ relative to the nucleus. The mean observational value of the scaleheight obtained $\left(\left\langle h_{z}\right\rangle=0\right.$.' $\left.^{\prime} 125\right)$ is the same as for exponential objects (Sect. 3.3) with a mean dispersion of individul values of 0. '03. Taking into account that the redshift and angular diameter distributions in both subsamples are approximately the same, one can conclude that non-exponential disks must have approximately the same corrected value of the scale height $\left(h_{z} \sim 0.5 \mathrm{kpc}\right)$.

\section{Discussion and conclusions}

Our sample of distant edge-on galaxies shows an unexpectedly large fraction of non-exponential disks. From this observation we infer that many sub- $L^{*}$ disks are still in the formation process at $z \sim 1$, or the degree of tidal perturbations is higher, which has already been observed (e.g. Le Févre et al. 2000). One of the possible mechanisms to redistribute matter and to develop the exponential profile is the action of a long-lived bar (e.g. Combes et al. 1990). The young galaxies of our sample may not have experienced enough bar torques for this process, either because of very transient bars in very unstable disks, or because the effects of bars are continuously perturbed by mergers and accretion. The consequence is that a galaxy will spend much less of its time in a barred state. This conclusion is in good agreement with the general depletion of barred spirals at $z>0.7$ (Abraham et al. 1999; van den Bergh 2002).
We have taken into account the effect of the PSF to demonstrate that the distant disks examined in this work are thicker by a factor of 1.5 if compared to local disk sample. This is a preliminary result based on a small number of sub- $L^{*}$ galaxies, and should be confirmed by larger statistical samples.

Since the star formation rate increases strongly with redshift (e.g. Madau et al. 1996; Flores et al. 1999; Thomson et al. 2001) one could speculate that the fraction of young stars formed in the midplane of disks is expected to be much higher in the past, making the young disk appear much thinner. Previous work on local samples has already concluded that tidal interactions between galaxies have a certain thickening effect of the order of 1.5-2 (Reshetnikov \& Combes 1997; Schwarzkopf \& Dettmar 2000a). Since the frequency of galaxy interactions/mergers increases strongly with redshift, the present result fits into this picture. If one assumes that all disks have undergone such interactions, either additional cooling processes for the hot disks or a substantial growth predominantly in the radial direction are required to explain the flatness of disks in the local Universe.

However, other parameters should be taken into account in the interpretation. The distant disks are on absolute scales smaller than the local sample disks. It is likely that the morphological types are later (less evolved) and the masses of the galaxies smaller on average, as already found in previous studies based on HST surveys (e.g. Abraham et al. 1996; Glazebrook et al. 1998). The lower frequency of bars, associated with the smaller bulge-to-disk ratio, suggests that disks are more unstable and dynamically hotter (Abraham et al. 1999), which could explain the present thickening effect. In this context it is also noteworthy to mention that the galaxies of our sample contain only a few bulges as can be judged from the major axis profiles in Fig. 8. This can either be interpreted as evidence for a later formation of bulges by secular processes (e.g. Combes 2001) or that our sample is selected such that it represents early phases of late morphological types only.

Acknowledgements. VR acknowledges support by DAAD for his extended stay at Ruhr-University Bochum and by Russian Federal Program "Astronomy" (40.022.1.1.1101). We thank Michael Pohlen for his help improving the text. We would like to thank the anonymous referee whose detailed remarks have helped improve the paper.

\section{References}

Abraham, R. G., van den Bergh, S., Glazebrook, K., et al. 1996, ApJS, 107,1

Abraham, R. G., Merrifield, M. R., Ellis, R. S., Tanvir, N. R., \& Brinchmann, J. 1999, MNRAS, 308, 569

Barteldrees, A., \& Dettmar, R.-J. 1994, A\&AS, 103, 475

Binney, J. J., \& Lacey, C. G. 1988, MNRAS, 230, 597

Bizyaev, D., \& Mitronova, S. 2002, A\&A, 389, 795

Block, D., Bournaud, F., Combes, F., Puerari, I., \& Buta, R. 2002, A\&A, 394, L35

Bournaud, F., \& Combes, F. 2002, A\&A, 392, 83

Bottema, R. 1993, A\&A, 275, 16

Byun, Y.-I. 1992, Dust Opacity and Structure of Spiral Galaxies, Ph.D. Thesis (Austral. Nation. Univ., Canberra)

Byun, Y. I., Freeman, K. C., \& Kylafis, N. D. 1994, ApJ, 432, 114 
Combes, F. 2001, in Galaxy Disk and Disk Galaxies, ASP Conf. Ser., 230, 213

Combes, F., Debbasch, F., Friedli, D., \& Pfenniger, D. 1990, A\&A, 233,82

Dalcanton, J. J., \& Bernstein, R. A. 2002, AJ, 124, 1328

de Grijs, R. 1998, MNRAS, 299, 595

de Grijs, R., \& van der Kruit, P. C. 1996, A\&AS, 117, 19

de Jong, R. S. 1996, A\&A, 313, 377

de Vaucouleurs, G., de Vaucouleurs, A., Corwin, H. G., et al. 1991, The Third Reference Catalogue of Bright Galaxies (New York: Springer-Verlag)

Ferguson, H. C., Dickinson, M., \& Williams, R. 2000, ARA\&A, 38, 667

Fernández-Soto, A., Lanzetta, K. M., \& Yahil, A. 1999, ApJ, 513, 34

Flores, H., Hammer, F., Thuan, T. X., et al. 1999, ApJ, 517, 148

Fontana, A., D'Odorico, S., Poli, F., et al. 2000, AJ, 120, 2206

Freeman, K. C. 1970, ApJ, 160, 811

Giovanell, R., Haynes, M. P., Salzer, J. J., et al. 1995, AJ, 110, 1059

Glazebrook, K., Abraham, R., Santiago, B., Ellis, R., \& Griffiths, R. 1998, MNRAS, 297, 885

Jiang, I-G., \& Binney, J. 1999, MNRAS, 303, L7

Keel, W. C., \& White, R. E. III. 2001, AJ, 122, 1369

Kregel, M., van der Kruit, P. C., \& de Grijs, R. 2002, MNRAS, 334, 646

Le Févre, O., Abraham, R., Lilly, S. J., et al. 2000, MNRAS, 311, 565

Lilly, S. J., Tresse, L., Hammer, F., et al. 1995, ApJ, 455, 108

Lilly, S. J., Schade, D., Ellis, R., et al. 1998, ApJ, 500, 75

Ma, J., Peng, Q-H., \& Gu, Q-S. 1998, A\&AS, 130, 449

Madau, P., Ferguson, H. C., Diskinson, M. E., et al. 1996, MNRAS, 283,1388

Matthews, L. D., \& Wood, K. 2001, ApJ, 548, 150

Misiriotis, A., Kylafis, N. D., Papamastorakis, J., \& Xilouris, E. M. 2000, A\&A, 353, 117

Mo, H. J., Mao, S., \& White, S. D. M. 1998, MNRAS, 295, 319

Oke, J. B. 1974, ApJS, 27, 21
Quinn, P. J., Hernquist, L., \& Fullagar, D. P. 1993, ApJ, 403, 74

Reshetnikov, V. P., Hagen-Thorn, V. A., \& Yakovleva, V. A. 1993, A\&A, 278, 351

Reshetnikov, V. P., \& Combes, F. 1997, A\&A, 324, 80

Reshetnikov, V., Battaner, E., Combes, F., \& Jiménez-Vicente, J. 2002, A\&A, 382, 513 (Paper I)

Robin, A. C., Haywood, M., Creze, M., et al. 1996, A\&A, 305, 125

Sancisi, R., Broeils, A., Kamphuis, J., \& van der Hulst, T. 1990, in Dynamics and Interactions of Galaxies, ed. R. Wielen (Springer), 304

Schwarzkopf, U., \& Dettmar, R.-J. 2000a, A\&AS, 144, 85

Schwarzkopf, U., \& Dettmar, R.-J. 2000b, A\&A, 361, 451

Schwarzkopf, U., \& Dettmar, R.-J. 2001, A\&A, 373, 402

Serjeant, S., Dunlop, J. S., Mann, R. G., et al. 2002, MNRAS [astro-ph/0201502]

Thompson, R. I., Weymann, R. J., \& Storrie-Lombardi, L. J. 2001, ApJ, 546, 694

Toomre, A. 1990, in Dynamics and Interactions of Galaxies, ed. R. Wielen (Springer), 292

Toth, G., \& Ostriker, J. P. 1992, ApJ, 389, 5

Tully, R. B., Pierce, M. J., Huang, J.-S., et al. 1998, AJ, 115, 2264

van den Bergh, S. 2002, PASP, 114, 797

van den Bergh, S., Cohen, J. G., Hogg, D. W., \& Blandford, R. 2000, AJ, 120, 2190

van den Bergh, S., Cohen, J. G., \& Crabbe, Ch. 2001, AJ, 122, 611

van der Kruit, P. C., \& Searle, L. 1981, A\&A, 95, 105

van der Kruit, P. C., \& Searle, L. 1982, A\&A, 110, 61

Velazquez, H., \& White, S. D. M. 1999, MNRAS, 304, 254

Walker, I. R., Mihos, J. C., \& Hernquist, L. 1996, ApJ, 460, 121

Westera, P., Samland, M., Buser, R., \& Gerhard, O. E. 2002, A\&A, 389, 761

Wielen, R. 1977, A\&A, 60, 263

Williams, R. E., Blacker, B., Dickinson, M., et al. 1996, AJ, 112, 1335 